\title{
Importance des facteurs édaphiques dans la répartition des forêts subalpines d'adret sur serpentines, prasinites et gneiss en Val d'Aoste (Italie)
}

\author{
JP Verger \\ Laboratoire de biologie végétale, Faculté des sciences, 87060 Limoges Cedex, France
}

(Reçu le 20 novembre 1988; accepté le 27 avril 1989)

Résumé - Dans le contexte bioclimatique intra-alpin du Val d'Aoste, à l'étage subalpin, les serpentines, roches ultrabasiques, induisent le mélézin comme forêt climacique. Aux expositions chaudes on trouve le Junipero-Arctostaphyletum laricetosum (nouvelle sous-association Verger, 1987), en milieu semi-mésophile le Rhodoreto-Vaccinietum vitis-idaea laricetosum (variante nouvelle des serpentines Verger, 1987) et en milieu mésophile le Rhodoreto-Vaccinietum laricetosum défini par Ozenda (1983) dans les Alpes du Sud. Les sols sont des rankers ou des sols brunifiés faiblement ocreux. Dans le même temps les prasinites, roches basiques, évoluent comme les gneiss acides. La forêt climacique est représentée par la variante à Vaccinium vitis-idaea du Luzulo-luzulinae piceetum (Braun-Blanquet, 1936 in Braun-Blanquet et al, 1939) sur prasinites, par celle à Vaccinium myrtillus sur gneiss, dans le bas de l'étage. Ces 2 variantes se retrouvent au niveau du Rhodoreto-Vaccinietum cembretosum Braun-Blanquet du haut de l'étage. Les sols sont alors des sols podzoliques ou des podzols.

Val d'Aoste / subalpin / climax forestier / serpentines / sol brunifié et mélézin / prasinites et gneiss / podzolisation / pessière et cembraie

Summary - Importance and role of edaphic determinism in the distribution of subalpine forests along warm slopes on serpentines, prasines and gneiss in Val d'Aoste (Italy). Because of the variety of rocks and different local climates in the Val d'Aoste subalpine zone, there is a large variety of vegetation.

On serpentine Junipero-Arctostaphyletum laricetosum (new association Verger, 1987) occurs on warmer slopes. Neutral acidophilous species grow on ranker and brown soils. On cooler slopes, Larix decidua reaches its ultimate stage of development. Rhodoreto-Vaccinietum laricetosum, with acidophilous species but without bryophytes, grows on brown soils where humus is a moder-mor.

At the lower limits of the subalpine zone, Piceetum subalpinum occurs along slopes on prasines and gneiss, while Rhodoreto-Vaccinietum Cembretosum grows along on the upper zone limits. On serpentine, where hydroxyferric complexes and magnesium prevail, lower acidity, permeability and soil dryness are favourable for Larix. Podzolic soils, where acidophilous organic matter, aluminium and protons prevail are favourable for Picea and Pinus cembra.

Aosta valley / subalpine zone / forest climax / serpentines / Larix decidua forest and brown soils / prasines and gneiss / podzolic soils / Picea and Pinus cembra forests 


\section{INTRODUCTION}

L'étude de la végétation arborée conduit à la distinction de 6 séries principales de végétation (Ozenda, 1983) à l'étage subalpin des Alpes:

- les séries subalpines de l'épicéa et du sapin occupent le subalpin inférieur.

Trois séries caractérisent surtout les massifs préalpins, sans que leurs aires se superposent:

- la série du pin mugo (Pinus mugo Turra), bien représentée dans les préalpes orientales nord et sud ainsi que dans l'axe intra-alpin, devient sporadique dans les Alpes orientales;

- la série préalpine du pin à crochet (Pinus uncinata Miller) occupe les préalpes de Suisse occidentale, de Savoie et du Dauphiné;

- une série altiméditerranéenne, presque asylvatique, apparaît en Haute-Provence et dans les Alpes ligures.

Une parenté écologique: relative xérophilie, plateaux et crêtes calcaires, unit ces séries.

- Le subalpin de l'axe interne est occupé par le pin cembro (Pinus cembra L.) et le mélèze (Larix decidua Miller).

Les vallées du versant sud des Alpes Pennines, en Val d'Aoste (fig. 1) appartiennent à la zone intra-alpine. La forêt se développe sur des substrats variés allant des roches ultrabasiques aux roches acides, en passant par les roches basiques. Comme ces affleurements se retrouvent souvent côte à côte dans ces vallées, le facteur climatique agit de façon identique sur la couverture forestière. C'est donc le facteur édaphique, et à travers lui la nature de la roche-mère, qui influe sur la composition du tapis végétal.

Nous nous proposons donc de rechercher comment, et dans quelle mesure, ce facteur agit. La présence des serpentines d'une part, des prasinites d'autre part, dont la pédogenèse est peu connue ou inconnue en milieu de montagne, constitue également une motivation importante de l'étude.

\section{LES MÉTHODES D'ANALYSE}

Pour chaque relevé de végétation, nous avons établi une fiche stationnelle précise regroupant, outre les données classiques (altitude, exposition, pente, position topographique, recouvrement végétal...), les conditions microstationnelles spécifiques, avec une attention particulière pour l'alimentation hydrique.

\section{Méthodes appliquées à la végétation}

Les relevés floristiques sont effectués selon la méthode phytosociologique classique définie par Braun-Blanquet (1964). Les relevés retenus résultent d'un tri. Après échantillonnage révélant les principales variations (topographie, sylvofaciès, types de sols), effectué en fonction aussi des possibilités de parcours, nous avons retenu les relevés les plus représentatifs des grands types forestiers. En ce qui concerne les serpentines, nous avons pu suivre la colonisation forestière des éboulis et le cheminement vers la forêt adulte. La construction des tableaux floristiques résulte d'une opération manuelle. Par contre, la classification des espèces en groupes écologiques provient d'un tri statistique préalable. Les données bibliographiques et surtout nos propres analyses nous ont amené à proposer une classification nouvelle des espèces (Verger, 1987 et 1989b), déterminée à partir:

- du rapport entre l'acidité d'échange $\left(\mathrm{Ae}=\mathrm{A} \mathrm{l}^{3+}\right.$ $+\mathrm{H}^{+}$) et la somme (S) des bases échangeables d'une part;

- de l'économie de l'eau d'autre part (fig. 2).

\section{Méthodes utilisées sur les échan- tillons de sol (fraction 0-2 $\mathrm{mm}$ )}

La granulométrie est réalisée après destruction de la matière organique par l'hypochlorite de sodium, mise en suspension des argiles par action des résines $\mathrm{Na}^{+}$échangeuses d'ions (Rouiller et al, 1981) et prélèvement à la pipette de Robinson des diverses fractions. 


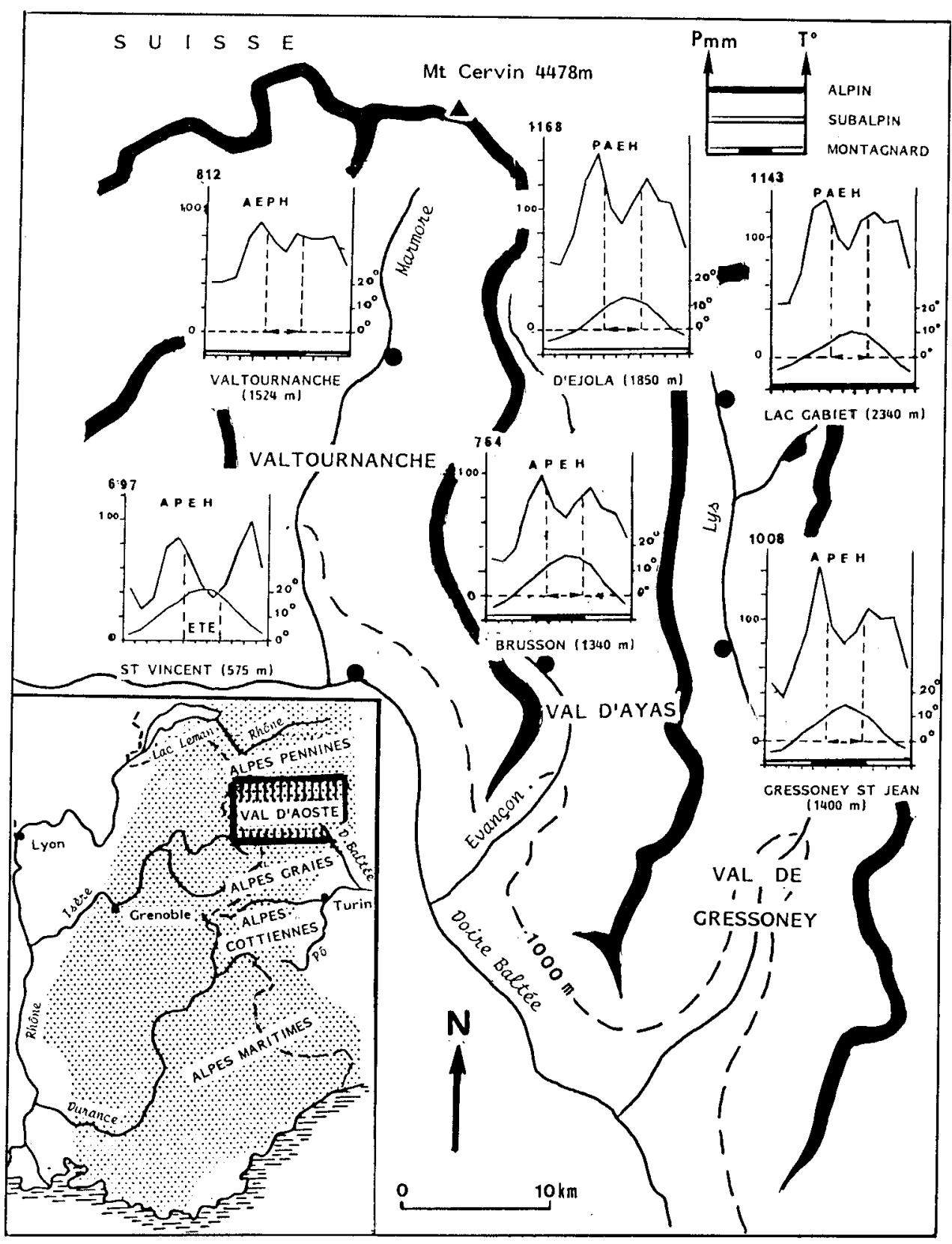

Fig. 1. Climatologie. Diagrammes ombrothermiques des vallées de Valtournanche, Val d'Ayas et Val de Gressoney (Val d'Aoste, Italie) 


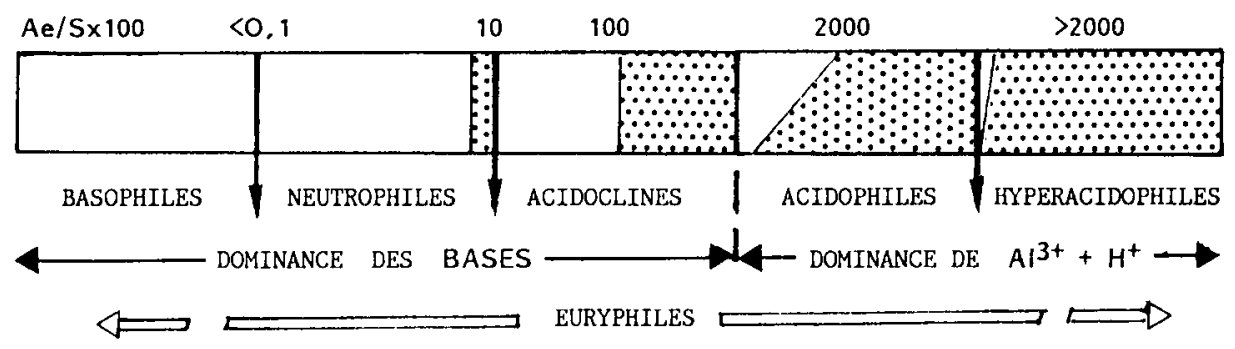

a - Influence des ions de 1'acidité.

\begin{tabular}{|c|c|c|c|c|}
\hline XEROPHILES & $\begin{array}{c}\text { XERO } \\
\text { MESOPHILES }\end{array}$ & $\begin{array}{c}\text { MESO } \\
\text { XEROPHILES }\end{array}$ & MESOPHILES & $\begin{array}{c}\text { MESO } \\
\text { HYGROPHILES }\end{array}$ \\
\hline
\end{tabular}

Eau - Exposition - Granulométrie - Drainage - Topographie ...

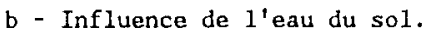

Fig. 2. Répartition synoptique des espèces végétales en fonction des ions de l'acidité et de l'eau du sol.

$\left.\begin{array}{ll}1 & \text { Brusson } \\ 2 & \text { Champoluc }\end{array}\right\}$ VAL D'AYAS

$\left.\begin{array}{l}3 \text { Valtournanche } \\ 4 \text { Lac Goillet }\end{array}\right]$ VALTOURNANCHE

5 Gressoney St Jean

6 Gressoney Trinité

7 d'Ejola

8 Lac Gabiet

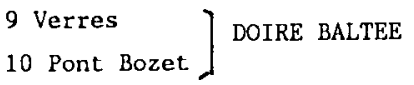

11 Lémie

ALPES GRAIES

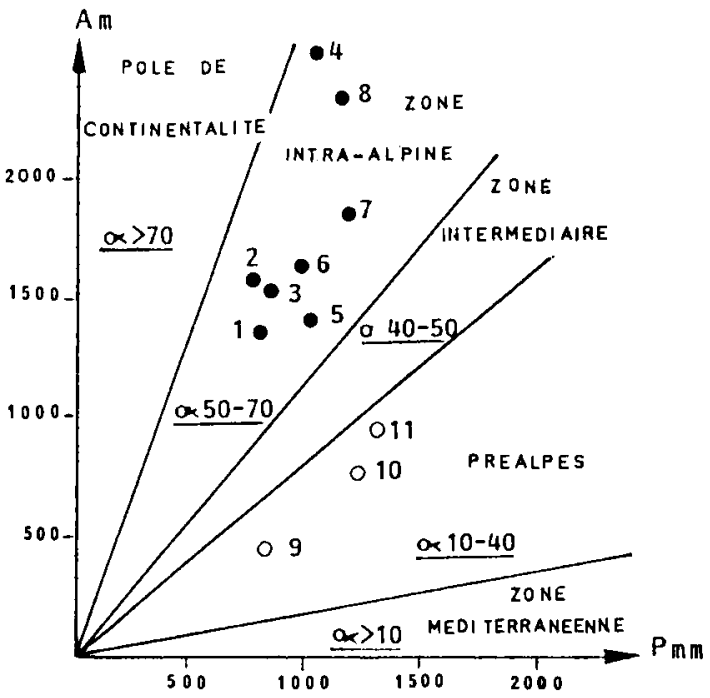

Fig. 3. Climatologie: Indice de continentalité hydrique de GAMS (cotgte o Pmm/Am).

La mesure électrométrique du $\mathrm{pH}$ est effectuée sur un mélange sol/solution de rapport $2 / 5$. La lecture du $\mathrm{pH}$ est effectuée sur le surnageant, après mise en contact de 4 heures $(\mathrm{pH}$ eau), ou après agitation rotative de $1 \mathrm{~h}(\mathrm{pH} \mathrm{KCl})$.
Les bases échangeables sont extraites par percolation à l'acétate d'ammonium tamponné à $\mathrm{pH} 7$ pour $\mathrm{K}^{+}$et $\mathrm{Na}^{+}$, par une solution de $\mathrm{KCl}$ normal pour $\mathrm{Ca}^{2+}, \mathrm{Mg}^{2+}$ et les ions de l'acidité $(A)^{3+}$ et $\left.\mathrm{H}^{+}\right)$. Les protons sont dosés par titrimé- 
trie, tous les autres éléments par spectrophotométrie d'absorption atomique (Spectromètre Atomspek Hilger-Watts $\mathrm{H} 1170$ ).

La sommation des bases échangeables évaluées à $\mathrm{pH} 7$ (T7) et des acidités ( $\mathrm{Ae}$ ) donne la capacité totale d'échange (T) au pH du sol.

Les éléments amorphes (fer et aluminium) sont extraits par le réactif de TAMM (T), et les formes amorphes et oxydes par le réactif de Mehra-Jackson (MJ). Le dosage se fait par absorption atomique.

Le carbone organique est dosé au Carmograph 8 , par combustion de la matière organique à $950^{\circ} \mathrm{C}$ dans un courant d'oxygène en excès et mesure des variations de conductivité électrique d'une solution de soude N/25 par le $\mathrm{CO}_{2}$ dégagé.

Pour le dosage de l'azote, les échantillons sont minéralisés à $900^{\circ} \mathrm{C}$ (Buchi 425) en milieu sulfurique concentré en présence de catalyseur. Le dosage final est réalisé selon la méthode de Kjeldahl.

\section{LE CLIMAT}

Les vallées de Gressoney, Val d'Ayas et Valtournanche font partie du secteur intra-alpin, à sécheresse relative. L'indice de continentalité hydrique de Gams (1950), qui exprime la valeur de la cotangente de l'angle de continentalité mesuré par le rapport des précipitations et de l'altitude ( $P$ en $\mathrm{mm} / \mathrm{A}$ en $\mathrm{m}$ ), est compris entre 50 et 70 (Fig. 3).

L'examen des courbes ombro-thermiques (Fig. 1) des stations situées à la base (Brusson, d'Ejola, Gressoney Saint-Jean) ou en limite supérieure de l'étage subalpin (Gabiet) ne montre aucune période de sécheresse $(\mathrm{P}=2 \mathrm{~T})$ ou de subsécheresse ( $P=3 T$ ) (Rey, 1960). La xéricité de la basse vallée (Saint-Vincent) n'atteint pas le subalpin, comme c'est le cas en Briançonnais (Cadel, 1980). D'une façon globa- le, la faiblesse des précipitations ne favorisera pas les processus de podzolisation.

\section{LES ROCHES MÈRES}

Leur composition chimique moyenne est rappelée dans le tableau I. Les gneiss acides représentent le pôle siliceux $(70 \%)$ riche en alumine $\left(14 \%\right.$ d'Al$\left._{2} \mathrm{O}_{3}\right)$, mais pauvre en fer, calcium et magnésium dont le rôle est primordial dans les processus de podzolisation. Les prasinites basiques, appauvries en silice $(50 \%)$ constituent un pôle alumino-calcique $\left(16 \%\right.$ d'Al ${ }_{2} \mathrm{O}_{3}$ et $9 \%$ de $\mathrm{CaO})$, riche en fer $(9 \%)$, qui fournit à la pédogenèse des minéraux phylliteux (type 2-1) chloriteux. Les serpentines, particulièrement pauvres en silice $(40 \%)$, représentent le pôle ultra-basique ferro-magnésien, dépourvu d'aluminium (cation essentiel de l'argilogenèse) et calcium, avec l'antigorite comme phyllite. La proportion très variable de fer, aluminium et calcium, les énormes différences en magnésium constituent autant de différences fondamentales dans l'orientation de la pédogenèse.

\section{LES ESSENCES FORESTIÈRES DU SUBALPIN D'ADRET}

\section{Les caractères de l'étage en Val d'Aoste}

La limite inférieure du subalpin d'adret s'établit à $1550 \mathrm{~m}$ sur gneiss et prasinites, mais s'élève à $1700 \mathrm{~m}$ sur serpentinites en raison de la teinte foncée de la roche et des sols qui captent et emmagasinent mieux la chaleur solaire. La limite supérieure de la forêt dépasse de $200 \mathrm{~m}$ celle observée, à la même latitude, dans les

Tableau I. Composition chimique comparée des roches mères.

\begin{tabular}{|l|lllllllll|}
\hline \multicolumn{1}{|c|}{ ROCHE } & $\mathrm{SiO}_{2}$ & $\mathrm{Al}_{2} \mathrm{O}_{3}$ & $\mathrm{Fe}_{2} \mathrm{O}_{3}$ & $\mathrm{MnO}$ & $\mathrm{MgO}$ & $\mathrm{CaO}$ & $\mathrm{Na}_{2} \mathrm{O}_{3}$ & $\mathrm{~K}_{2} \mathrm{O}$ & $\mathrm{TiO}_{2}$ \\
\hline GNEISS & 71,55 & 13,9 & 3,01 & 0,06 & 1,04 & 0,69 & 4,10 & 3,01 & 0,36 \\
PRASINITES & 49 & 16,3 & 9 & 0,14 & 7,05 & 8,35 & 3,65 & 0,33 & 1,3 \\
SERPENTINES & 71,98 & 2,89 & 9,09 & 0,06 & 34,64 & 2,57 & 0,19 & 0,06 & - \\
\hline
\end{tabular}


zones externes des Alpes (Richard et Tonnel, 1987). Cette limite (autour de $2100 \mathrm{~m}$ ) plus homogène traduit l'égalisation climatique, y compris pédoclimatique, que l'on observe en se rapprochant de l'étage alpin de ces vallées. Les landines subalpines du Cetrario-Loiseleurietum atteignent $2500 \mathrm{~m}$.

Sur les fortes pentes des serpentines, le mélézin joue un rôle protecteur de premier ordre et l'essence constitue l'arbre clé de la forêt. Partant de la rhodoraie asylvatique et/ou du Junipero-Arctostaphyletum, le mélèze couvre tout le subalpin pour venir se mêler à Pinus sy/vestris au sommet de l'étage montagnard. L'influence anthropique apparaît faible (pas ou peu de souches coupées).

Sur prasinites et sur gneiss, le mélèze se cantonne dans la partie supérieure de la forêt et ne constitue plus qu'une essence de transition. Sur ces pentes moins fortes, la forêt résulte souvent d'une reforestation souvent liée à la déprise agricole (Lyabel, 1962). Par souci de comparaison, nous avons délaissé ces bois et retenu dans notre étude les forêts les plus âgées, développées sur pentes plus fortes. L'influence humaine passée est partout présente (souches, charbons de bois). Au contact de la lande à rhododendrons, la cembraie peut remplacer tout ou partie du mélézin, alors que, vers le bas, la pessière couvre le subalpin moyen et inférieur.

\section{La végétation forestière du subalpin}

\section{Sur roche mère ultrabasique (tableau II)}

Le mélèze succède soit à la lande à genèvrier nain des adrets, soit au rhododendron des expositions fraîches. Le sous-bois permet d'y reconnaître plusieurs stades d'évolution. Dans les premiers stades de colonisation des éboulis, où la strate herbacée laisse de nombreux vides, la régénération est active. Les espèces neutrophiles (Thymus praecox, Carline acaulis, Sesleria albicans, Thlaspi alpinum, Laserpitium latifolium) partagent le recouvrement avec les acidoclines (Festuca luedii, Lotus corniculatus,

Dactylorhiza sambucina) et les acidophiles (Festuca gr. ovina, Hieracium murorum, Deschampsia flexuosa). Les myrtilles sont absentes ou rares en sous-bois. En l'absence totale du pin cembro et du pin à crochet, ce type de forêt nous paraît devoir s'intégrer dans une sousassociation nouvelle à mélèze du Juniperion nanae que nous proposons d'appeler Junipero-Arctostaphyletum laricetosum.
Le mélézin du haut de l'étage montre une dominance presque totale des espèces de la lande. Les espèces neutrophiles et le genévrier nain sont sporadiques, les acidoclines rares. Le sous-bois comporte un tapis d'espèces acidophiles mésophiles (Rhododendron ferrugineum, Vaccinium uliginosum, Vaccinium myrtillus, Vaccinium vitis-idaea) et mésohygrophiles (Homogyne alpina). Cette rhodoraie sylvatique sur serpentines s'individualise par l'absence ou la rareté de l'épicéa, du pin cembro qui n'apparaissent que sous la forme de plantules tortueuses dès le quatrième ou cinquième verticille de branches. Cette torêt constitue un ensemble stable, climacique, où Carex fimbriata singularise le substrat serpentinique (Verger, 1983 et Richard J.L., 1985). Selon le degré d'humidité, on y distingue un Rhodoreto-Vaccinietum vitis-idaea laricetosum (par analogie avec les pessières) semi-mésophile et un Rhodoreto-Vaccinietum laricetosum mésophile récemment défini par Ozenda (1981).

\section{La pessière des prasinites et des gneiss (tableau III)}

Les 2 types de roches portent, dans le bas de l'étage, une forêt dominée par l'épicéa. Le mélèze est rare et sa présence transitoire. Avec la déprise agricole, on assiste à une recolonisation rapide par l'épicéa des mélézins existants.

Les pessières observées se caractérisent par la présence presque exclusive des espèces acidophiles. L'abondance et la dominance des vacciniées, la présence d'espèces différentielles comme Orthilia secunda, Deschampsia flexuosa, Hieracium murorum, Melampyrum sylvaticum, Luzula sylvatica et L. Luzulina, Saxifraga cuneifolia, Homogyne alpina, Geranium sylvaticum, Lonicera nigra, Cetraria islandica, apparentent ces pessières au groupement du Luzulo-luzulinae Piceetum (ou Piceetum subalpinum) Braun-Blanquet, 1936. L'homologie n'est cependant pas parfaite entre les forêts recouvrant les 2 roches.

La quasi-totalité des relevés de la pessière développée sur prasinites, par la forte présence de Vaccinium vitis-idaea, Deschampsia flexuosa, Anthoxantum odoratum, s'apparente à la variante vaccinietosum vitis-idaea décrite par Braun-Blanquet et al, en Engadine (1954). L'abondance de Vaccinium myrtillus et de Pinus cembra notamment l'éloigne des pessières xérophiles d'adret de Maurienne (Bartoli, 1966) et plus encore de celles de mode sec de Tarentaise (Gensac, 1964). 
Tableau II. La forêt subalpine sur serpentines (Val d'Aoste, Italie).

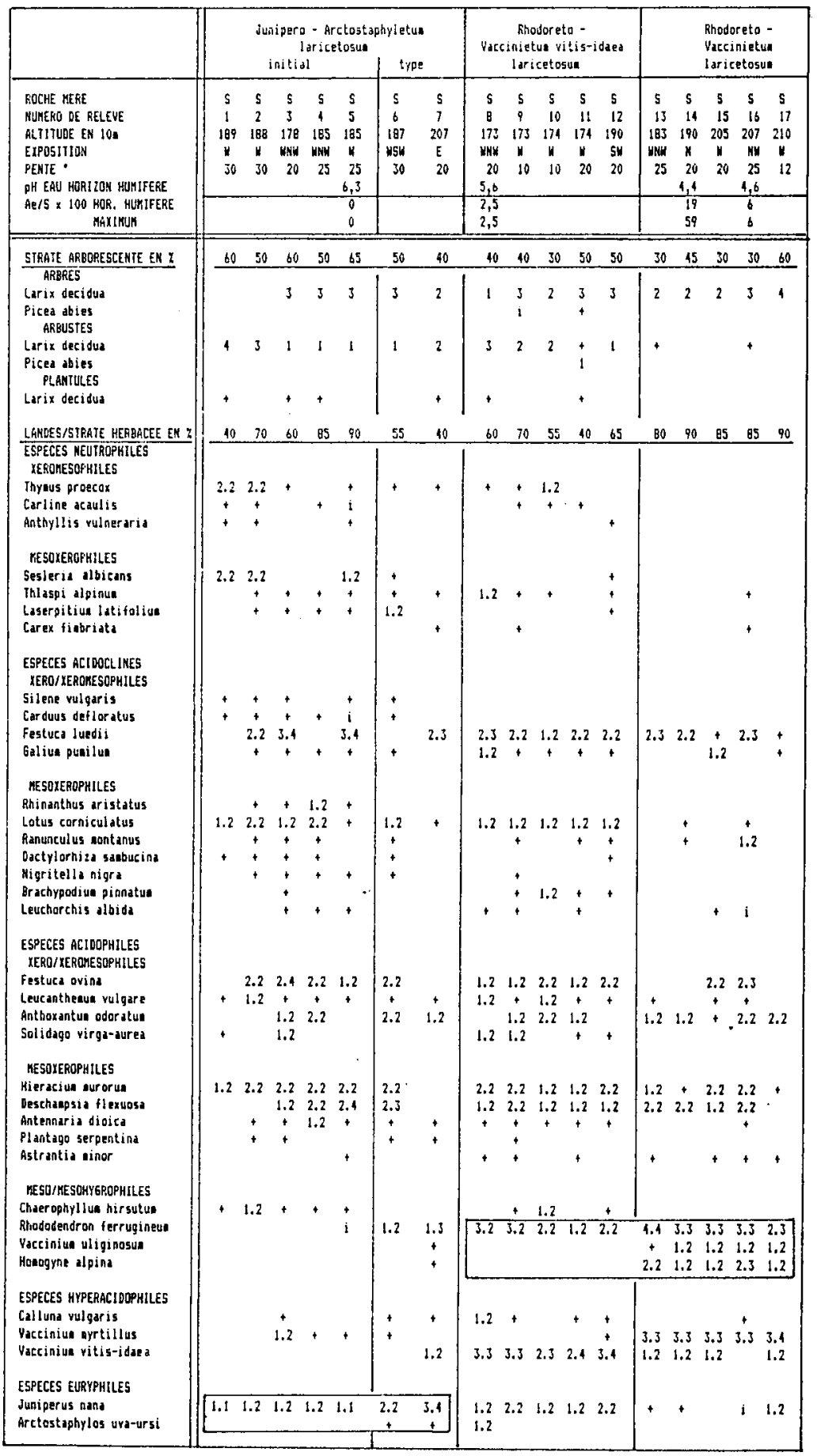


Tableau III. La forêt subalpine sur prasinites et sur gneiss (Val d'Aoste, Italie).

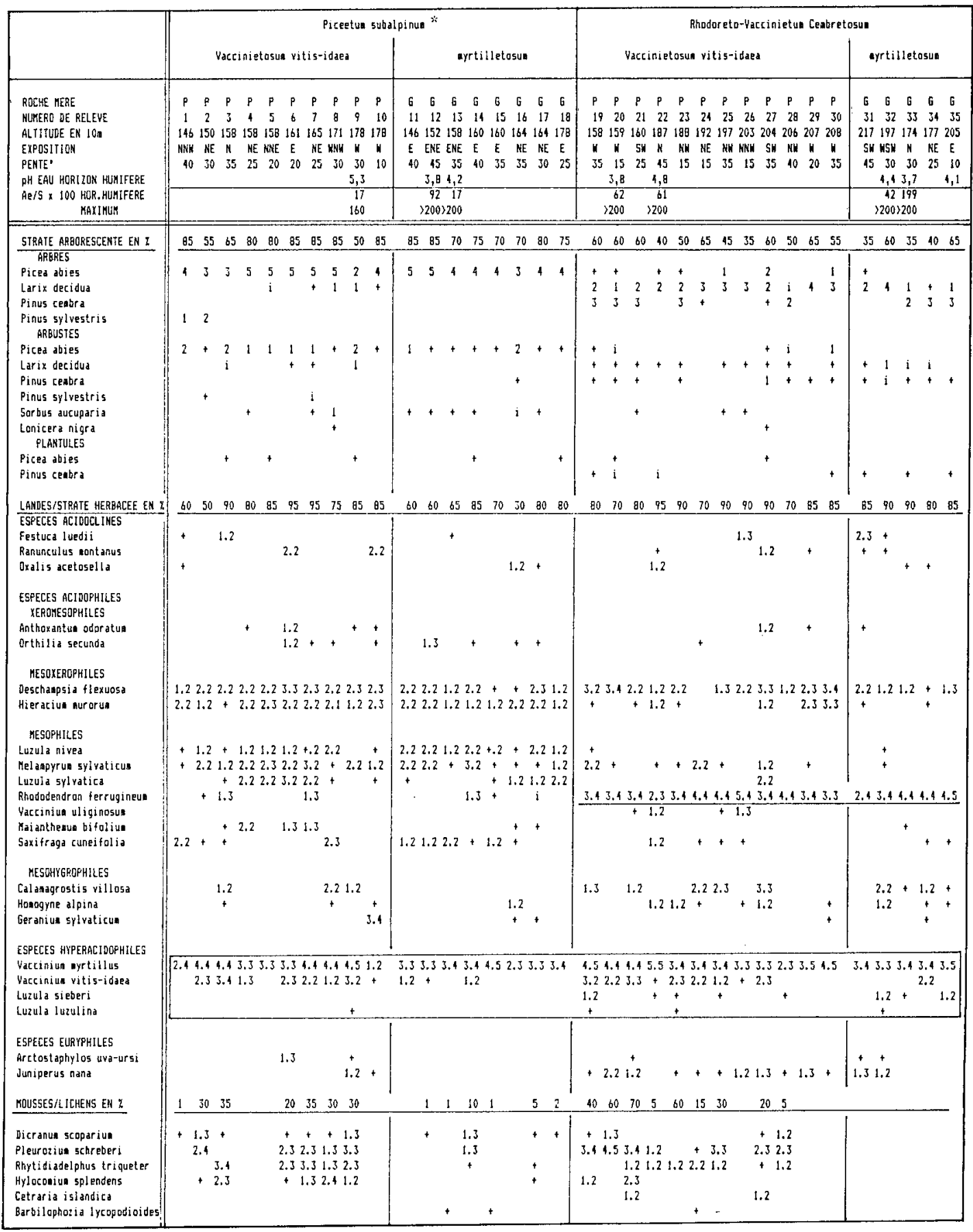

$*$ Picectun subalpinum $=$ Luzulo-luzulinae Piceetun 
Dans leur ensemble les relevés de la pessière sur gneiss acides, par la dominance de Vaccinium myrtillus, l'abondance de Luzula sylvatica, Melampyrum sylvaticum, la constance de Sorbus aucuparia et l'absence du mélèze se rapportent à la variante myrtilletosum Mayer, 1958 du Piceetum. Ils en diffèrent surtout par l'absence de Listera cordata.

\section{La cembraie sur prasinites et gneiss (tableau III)}

Comme la pessière, la cembraie se rencontre sur les 2 types de roches. Elle constitue dans tous les cas une évolution de mélézin ancien vers le climax à pin cembro. Le mélèze se pré-

Tableau IV. Propriétés physiques, matière organique, distribution des oxydes de fer et d'aluminium le long des profils.

\begin{tabular}{|c|c|c|c|c|c|c|c|c|c|c|c|c|c|c|c|c|}
\hline $\begin{array}{l}R \\
0 \\
C \\
H \\
E\end{array}$ & $N^{*}$ & $\begin{array}{l}\text { NIVEAU DE } \\
\text { PRELEVEMENT }\end{array}$ & $\begin{array}{c}\text { HUMI } \\
\text { DITE } \\
105^{\circ} \\
y\end{array}$ & $\begin{array}{c}\text { Pierro } \\
\text { site } \\
x\end{array}$ & $\begin{array}{r}56 \\
7 .\end{array}$ & $\begin{array}{r}5 f \\
z .\end{array}$ & $\begin{array}{l}16 \\
\text { \%. }\end{array}$ & $\begin{array}{l}\text { Lf } \\
\text { L. }\end{array}$ & $\begin{array}{l}\text { A } \\
\text { h. }\end{array}$ & $\begin{array}{l}\text { MATIE } \\
\qquad \\
h .\end{array}$ & $\begin{array}{l}N \\
Z .\end{array}$ & $C / N$ & $\begin{array}{r}E L \\
\quad \\
\end{array}$ & $\begin{array}{l}\text { LEMENTS } \\
\text { Fer } \\
\text { H. } \\
\text { HJ }\end{array}$ & $\begin{array}{l}\text { LIBR } \\
\text { Al } \\
\text { en }\end{array}$ & ES \\
\hline $\begin{array}{l}S \\
E \\
A \\
P \\
E \\
N \\
T \\
I \\
H \\
E\end{array}$ & 14 & $\begin{array}{ll}\text { AL } & 0-10 \\
\text { ALC } & 10-60 \\
\text { C } & 60-90 \\
\text { AOA1 } & 0-4 \\
\text { AL2 } & 4-14 \\
\text { Bh } & 14-22 \\
\text { B5 } & 22-50 \\
\text { IIC } & 50\end{array}$ & $\begin{array}{l}2,2 \\
1 \\
0,7 \\
8 \\
2,7 \\
2,5 \\
1,8 \\
1,2\end{array}$ & $\begin{array}{r}61 \\
58 \\
67 \\
6 \\
30 \\
6 \\
37 \\
35\end{array}$ & $\begin{array}{l}176 \\
154 \\
297 \\
323\end{array}$ & $\begin{array}{l}247 \\
250 \\
299 \\
333\end{array}$ & $\begin{array}{r}54 \\
94 \\
141 \\
104\end{array}$ & $\begin{array}{l}243 \\
243 \\
108 \\
111\end{array}$ & $\begin{array}{l}82 \\
96 \\
49 \\
35\end{array}$ & $\begin{array}{l}18,7 \\
10,9 \\
5 \\
\\
211,7 \\
39 \\
19,8 \\
5,8 \\
3,5\end{array}$ & $\begin{array}{l}0,61 \\
0,39 \\
0,23 \\
\\
6,27 \\
1,34 \\
1,21 \\
0,56\end{array}$ & $\begin{array}{l}30,6 \\
27,6 \\
21,3 \\
33,7 \\
29 \\
16,4 \\
10,3\end{array}$ & $\begin{array}{l}2,7 \\
2,4 \\
3 \\
3,6 \\
6,2 \\
7 \\
3,1 \\
5,4\end{array}$ & $\begin{array}{l}5 \\
5,8 \\
4,9 \\
\\
6,8 \\
10,1 \\
12,6 \\
21,1 \\
15,4\end{array}$ & $\begin{array}{l}0,4 \\
0,6 \\
0,4 \\
1,4 \\
1,1 \\
1,5 \\
1,3 \\
1,1\end{array}$ & $\begin{array}{l}1,5 \\
1,2 \\
1,8 \\
3 \\
2,7\end{array}$ \\
\hline $\begin{array}{l}\text { P } \\
R \\
\text { A } \\
5 \\
\text { I } \\
H \\
I \\
T \\
\text { E } \\
\text { S }\end{array}$ & 22 & $\begin{array}{lc}A O A 1 & 0-12 \\
A 12 & 12-27 \\
B h & 27-42 \\
B s & 42-62 \\
\text { IIC } & 62 \ldots \\
& \\
A O A 1 & 0-12 \\
A 2 & 12-20 \\
B h & 20-35 \\
B s & 35-80 \\
B / C & B 0 . . .\end{array}$ & $\begin{array}{c}30,8 \\
3,8 \\
7,2 \\
5,7 \\
1\end{array}$ & $\begin{array}{l}39 \\
34 \\
33 \\
28 \\
40\end{array}$ & $\begin{array}{l}265 \\
326 \\
300 \\
271 \\
253\end{array}$ & $\begin{array}{l}291 \\
348 \\
399 \\
402 \\
351\end{array}$ & $\begin{array}{r}135 \\
92 \\
86 \\
61 \\
\\
36 \\
62 \\
57 \\
66 \\
63\end{array}$ & $\begin{array}{r}215 \\
39 \\
93 \\
150 \\
15 \\
73 \\
60 \\
63 \\
70\end{array}$ & $\begin{array}{l}35 \\
18 \\
20 \\
18 \\
10\end{array}$ & $\begin{array}{c}282,5 \\
50 \\
30,2 \\
13,1 \\
1 \\
\\
76,4 \\
17,3 \\
14,7 \\
9,3 \\
3,1\end{array}$ & $\begin{array}{l}9,10 \\
1,59 \\
0,94 \\
0,49\end{array}$ & $\begin{array}{l}31,2 \\
31,4 \\
31,9 \\
26,7\end{array}$ & $\left\{\begin{array}{l}1,4 \\
2,1 \\
12 \\
10 \\
1,4 \\
1,4 \\
1,2 \\
4 \\
3,6 \\
1,2\end{array}\right.$ & $\begin{array}{c}2,5 \\
8,1 \\
21,4 \\
22 \\
5,8 \\
\\
3,2 \\
3 \\
7,8 \\
6,8 \\
3\end{array}$ & $\begin{array}{l}0,35 \\
0,5 \\
4,9 \\
3,5 \\
0,35 \\
1,5 \\
1,5 \\
4 \\
3,8 \\
2,4\end{array}$ & $\begin{array}{l}1 \\
1,7 \\
11,9 \\
0,7 \\
0,7 \\
\\
2,2 \\
2 \\
5,8 \\
5,8 \\
3\end{array}$ \\
\hline $\begin{array}{l}6 \\
N \\
\text { E } \\
I \\
5 \\
5\end{array}$ & 12 & $\begin{array}{ll}A 0 A 1 & 0-7 \\
A 2 & 7-11 \\
B h & 11-18 \\
B s & 18-25 \\
B / C & 25 \ldots \\
& \\
A 1 & 0-7 \\
A 2 & 7-15 \\
B h & 15-20 \\
B h / B s & 20-40 \\
B 5 & 10-50 \\
C & 50 \ldots\end{array}$ & $\begin{array}{l}5 \\
2,9 \\
2,7 \\
3,8 \\
2,6 \\
2,4\end{array}$ & $\begin{array}{l}30 \\
39 \\
34 \\
37 \\
40 \\
60\end{array}$ & $\begin{array}{l}164 \\
248 \\
222 \\
334 \\
336 \\
390\end{array}$ & $\begin{array}{l}370 \\
290 \\
314 \\
296 \\
348 \\
332\end{array}$ & $\begin{array}{l}57 \\
82 \\
85 \\
95 \\
63 \\
98\end{array}$ & $\begin{array}{r}144 \\
171 \\
168 \\
110 \\
120 \\
73\end{array}$ & $\begin{array}{l}65 \\
75 \\
78 \\
40 \\
35 \\
18\end{array}$ & $\begin{array}{r}94,5 \\
32,4 \\
25,3 \\
11,7 \\
12,8 \\
6,7\end{array}$ & $\begin{array}{l}3,78 \\
1,14 \\
0,74 \\
0,58 \\
0,57\end{array}$ & $\begin{array}{l}25 \\
28,4 \\
34,4 \\
22 \\
20,6\end{array}$ & $\begin{array}{l}1 \\
1,4 \\
1,9 \\
4,2 \\
1,6 \\
1,4 \\
0,9 \\
1,5 \\
3 \\
1,2 \\
2,5\end{array}$ & $\begin{array}{l}2,2 \\
3,4 \\
4 \\
4,8 \\
4,4 \\
3,8 \\
5,5 \\
6,1 \\
9,9 \\
15,5 \\
8,3\end{array}$ & $\begin{array}{l}1,2 \\
1,8 \\
2,3 \\
3,6 \\
6,8 \\
4,9\end{array}$ & $\begin{array}{l}1,4 \\
1,9 \\
2,3 \\
3,6 \\
6,8 \\
5\end{array}$ \\
\hline
\end{tabular}

T. : Extraction au réactif de Tamm.

M.J. : Extraction au réactif de Mehra-Jackson. 
sente sous la forme de très gros arbres épars, à nombreux rameaux morts, et/ou de rares arbustes grêles. Le pin cembro, rarement adulte, sauf sur gneiss, se présente alors sous la forme de sujets vigoureux, très droits et de belle venue.

Dans les 2 cas, on retrouve, parmi les strates basses des landes et des herbacées, la dominance quasi absolue des espèces acidophiles. Les espèces mésophiles caractéristiques de l'association du Rhodoreto-Vaccinietum (Braun-Blanquet et al, 1954) sous-association Cembretosum: Pinus cembra, Rhododendron ferrugineum, Vaccinium myrtillus et vitis-idaea, Calamagrostis villosa sont constantes et/ou dominantes. Les mousses sont largement représentées sur prasinites: Pleurozium schreberi, Dicranum scoparium, Hylocomium splendens dans les parties les plus fraîches, alors que Rhytidiadelphus triqueter se limite aux plages un peu plus ensoleillées.

Comme pour la pessière, des nuances séparent les groupements sur prasinites et sur gneiss. Les premiers sont à rapprocher d'une variante à Vaccinium vitis-idaea, en raison de l'abondance de l'espèce. Sur les gneiss plus frais, et dans le haut de l'étage sur prasinites, c'est la variante myrtilletosum qui se développe.

\section{LES SOLS FORESTIERS DU SUBALPIN}

La profondeur très voisine des profils ne constitue pas un critère discriminant, de même que la pierrosité. Au sein de la terre fine, la microdivision affecte rapidement et plus fortement les roches ultrabasiques que l'ensemble prasinitesgneiss (tableau IV). II en résulte un milieu enrichi en éléments les plus fins sur serpentines et une mise à disposition plus importante pour la végétation des cations des minéraux primaires de la roche.

C'est ainsi (tableau $\mathrm{V}$ ) que le taux de saturation reste élevé, même à $\mathrm{pH} 7$, sur ces dernières roches, alors qu'il est très faible sur les gneiss. $\mathrm{Au} \mathrm{pH}$ du sol, les milieux sont eutrophes ou faiblement mésotrophes (selon la terminologie de Penel, 1979) sur ultrabasites, alors qu'une désaturation progressive des humus gagne la base des profils et s'accentue des prasinites (milieux mésotrophes à oligomésotrophes) aux gneiss (seulement mésotrophes au niveau de l'humus). Ces valeurs du $\mathrm{pH} \mathrm{KCl}$ mettent en valeur un aspect fondamental de la pédogénèse sur ultrabasites en montagne. Les protons, exceptionnels, l'aluminium, discret, n'induisent qu'une acidification réduite qui va jouer un rôle discriminant dans la répartition des espèces végétales en favorisant les neutrophiles et les acidoclines aux dépens des acidophiles. Ces résultats sont aussi la conséquence de la richesse des sols serpentiniques en magnésium d'une part (ce qui est classique sur ce type de roche), et de la richesse en calcium d'autre part, élément normalement très déficitaire dans la roche et très intensément concentré au sein de l'horizon humifère par le jeu du cycle biogéochimique. Nous reviendrons sur cet aspect, conséquence de la mise en place de la roche au cours de l'orogenèse alpine (Boillot, 1987), dans une prochaine note.

La redistribution (tableau IV) précoce de l'aluminium (Souchier, 1971), dès les stades acides de la brunification (Jeanroy, 1983), montre un double aspect:

- quantitatif, avec des taux très bas $(<1 \%$ sur serpentines), beaucoup plus élevés sur prasinites (jusqu'à $12 \%$ ) et gneiss (jusqu'à $7 \%$ );

- qualitatif, avec une redistribution nulle ou modérée sur ultrabasites $\left(K_{A l}=1,4\right)$, très importante sur les 2 autres roches $\left(K_{A P}>2\right)$, le maximum se situant en Bh sur prasinites, en Bs sur gneiss. $\left(\mathrm{K}_{\mathrm{Al}}=\right.$ teneur en $\mathrm{Al}$ de $\mathrm{B} /$ teneur moyenne proportionnelle entre $A 1$ et $C$ ).

En fait, les horizons Bs types se caractérisent surtout par une accumulation d'aluminium amorphe (Jeanroy, 1983). C'est ce que montrent les sols sur gneiss (et prasinites), nettement marqués par la podzolisation. Les serpentines ont, par contre, un comportement de sol brunifié, tant est discrète l'augmentation de l'aluminium amorphe en $\mathrm{Bh}$ et Bs.

Le fer (tableau IV) montre aussi une redistribution différenciée selon les roches. L'appauvrissement des horizons de surface et l'accumulation au sein des horizons spodiques (Bs) sont nets sur prasinites et gneiss alors qu'ils sont plus mesurés sur serpentines. La libération de cet élément, qui s'oppose à la podzolisation (Souchier, 1971; Robin, 1979), est plus élevée dans l'ensemble des profils sur ultrabasites, sous l'effet d'une altération biosphérique (en milieu aéré) des minéraux ferro-magnésiens synchrone d'une argilisation accrue (Jeanroy, 1983).

Au total, la dynamique du fer et de l'aluminium traduit des processus nets de redistribution et donc de podzolisation, sur prasinites et gneiss acides, alors qu'ils sont limités et largement liés à l'altération sur serpentines.

La biodégradation des humus est très ralentie et comparable dans les 3 cas. Au sein des horizons organo-minéraux la richesse en cations (bases et fer) accélère par contre 
Tableau V. Capacité totale d'échange ( $T$ ) et taux de saturation $(\mathrm{V}=\mathrm{S} / \mathrm{T})$, en meq pour $100 \mathrm{~g}$ de sol, à $\mathrm{pH} 7$ et au $\mathrm{pH}$ du sol.

\begin{tabular}{|c|c|c|c|c|c|c|c|c|c|c|c|c|c|c|c|c|}
\hline $\begin{array}{l}R \\
0 \\
C \\
H \\
E\end{array}$ & $\mathrm{H}^{\circ}$ & $\begin{array}{l}\text { NIVEAUL DE } \\
\text { PRELEVEMENT }\end{array}$ & $\mathrm{H} 2 \mathrm{O}$ & $\mathrm{KCl}$ & Catt & $\mathrm{Mg}++$ & $S$ ECH & $\mathrm{Nat}$ & $\mathrm{Mnt+}$ & $S$ & $\begin{array}{r}\text { ION } \\
\text { L'AC } \\
{ }^{A}{ }^{A}\end{array}$ & $\begin{array}{l}\text { DE } \\
\text { IDIJE } \\
\text { AlJ+ }\end{array}$ & 17 & V7 & $\begin{array}{c}\mathrm{T} \\
\text { au ph }\end{array}$ & sol \\
\hline $\begin{array}{l}S \\
E \\
R \\
P \\
E \\
N \\
T \\
I \\
N \\
E\end{array}$ & 14 & $\begin{array}{lc}\text { Al } & 0-10 \\
\text { AlC } & 10-60 \\
\text { C } & 60-90 \\
\text { AOA1 } & 0-4 \\
\text { A12 } & 1-14 \\
\text { Bh } & 14-22 \\
\text { BS } & 22-50 \\
\text { IIC } & 50\end{array}$ & $\begin{array}{l}6,30 \\
6,87 \\
7,28 \\
4,4 \\
5 \\
5,8 \\
6 \\
6,1\end{array}$ & $\begin{array}{l}6,25 \\
6,57 \\
6,68 \\
3,6 \\
3,74 \\
4,46 \\
4,82 \\
4,88\end{array}$ & $\begin{array}{l}2,8 \\
3,18 \\
2,47 \\
5,96 \\
1,87 \\
2,37 \\
3,56 \\
0,37\end{array}$ & $\begin{array}{l}1,29 \\
1,26 \\
0,72 \\
\\
9,24 \\
3,55 \\
1,71 \\
1,38 \\
0,71\end{array}$ & $\begin{array}{l}0,08 \\
0,03 \\
0,02 \\
\\
0,42 \\
0,08 \\
0,06 \\
0,02 \\
0,02\end{array}$ & $\begin{array}{l}0,17 \\
0,16 \\
0,21 \\
0,13 \\
0,20 \\
0112 \\
0,06 \\
0,13\end{array}$ & $\begin{array}{l}0,06 \\
0,04 \\
0,02 \\
0,2 \\
0,01 \\
0,16\end{array}$ & $\begin{array}{c}4,34 \\
4,63 \\
3,42 \\
15,75 \\
5,7 \\
7,26 \\
5,02 \\
1,23\end{array}$ & $\begin{array}{l}0,95 \\
0,60\end{array}$ & $\begin{array}{l}2,10 \\
2,80 \\
0,95 \\
0,45 \\
0,40\end{array}$ & $\begin{array}{l}6,8 \\
5,8 \\
3,45 \\
33,6 \\
12,8 \\
9,38 \\
5,3 \\
3,6\end{array}$ & $\begin{array}{l}66 \\
80 \\
99 \\
47 \\
44 \\
77 \\
95 \\
34\end{array}$ & $\begin{array}{l}4,47 \\
4,6 \\
3,4 \\
18,8 \\
9,1 \\
8,21 \\
5,47 \\
1,63\end{array}$ & $\begin{array}{l}84 \\
63 \\
88 \\
92 \\
75\end{array}$ \\
\hline $\begin{array}{l}P \\
R \\
A \\
S \\
I \\
H \\
I \\
T \\
E \\
S\end{array}$ & 22 & $\begin{array}{lr}\text { AOA1 } & 0-12 \\
\text { A12 } & 12-27 \\
\text { Bh } & 27-42 \\
\text { Bs } & 42-62 \\
\text { IIC } & 62 . . . \\
& \\
\text { AOA1 } & 0-12 \\
\text { A2 } & 12-20 \\
\text { Bh } & 20-35 \\
\text { Bs } & 35-80 \\
\text { B/C } & B 0 . .\end{array}$ & $\begin{array}{l}5,33 \\
4,16 \\
5,84 \\
6,13 \\
6,48 \\
\\
4,82 \\
5,02 \\
5,70 \\
5,85 \\
5,84\end{array}$ & $\begin{array}{l}3,29 \\
3,46 \\
1,47 \\
5,19 \\
5,08 \\
\\
3,81 \\
4,08 \\
4,60 \\
4,82 \\
4,73\end{array}$ & $\begin{array}{r}11,25 \\
1,53 \\
0,20 \\
0,32 \\
0,98 \\
\\
2,67 \\
0,06 \\
0,06 \\
0,05 \\
0,02\end{array}$ & $\begin{array}{l}1,46 \\
1,26 \\
0,91 \\
0,52 \\
1,20 \\
1,27 \\
0,36 \\
0,23 \\
0,14 \\
0,15\end{array}$ & $\begin{array}{l}1,38 \\
0,22 \\
0,13 \\
0,10\end{array}$ & $\begin{array}{l}0,21 \\
0,16 \\
0,16 \\
0,10\end{array}$ & 0,04 & $\begin{array}{r}15,71 \\
4,38 \\
1,49 \\
1,13 \\
2,02\end{array}$ & $\begin{array}{l}1,12 \\
1,39\end{array}$ & $\begin{array}{l}1,17 \\
5,78 \\
1,37 \\
0,30 \\
0,64 \\
\\
2,02 \\
3,33 \\
1,74 \\
1,20 \\
0,66\end{array}$ & $\begin{array}{l}50,6 \\
15,6 \\
13,7 \\
8,12\end{array}$ & $\begin{array}{l}31 \\
28 \\
11 \\
14\end{array}$ & $\begin{array}{r}18,34 \\
11,45 \\
2,86 \\
1,43 \\
2,66 \\
\\
6,4 \\
3,91 \\
2,04 \\
1,40 \\
0,84\end{array}$ & $\begin{array}{l}86 \\
38 \\
52 \\
79 \\
76 \\
\\
61 \\
11 \\
14 \\
14 \\
20\end{array}$ \\
\hline $\begin{array}{l}6 \\
N \\
E \\
I \\
S \\
S\end{array}$ & 12 & $\begin{array}{lc}\text { AOA1 } & 0-7 \\
\text { A2 } & 7-11 \\
\text { Bh } & 11-18 \\
\text { Bs } & 10-25 \\
\text { B/C } & 25 \ldots \\
& \\
\text { At } & 0-7 \\
\text { A2 } & 7-15 \\
\text { Bh } & 15-20 \\
\text { Bh/Bs } & 20-40 \\
\text { Bs } & 10-50 \\
\text { C } & 50 \ldots\end{array}$ & $\begin{array}{l}4,41 \\
3,82 \\
4,10 \\
4,91 \\
4,65 \\
4,82\end{array}$ & $\begin{array}{l}3,64 \\
3,64 \\
3,90 \\
4,34 \\
4,38 \\
4,32\end{array}$ & $\begin{array}{l}5,12 \\
0,31 \\
0,07 \\
0,05 \\
0,07 \\
0,15\end{array}$ & $\begin{array}{l}1,62 \\
0,22 \\
0,11 \\
0,05 \\
0,04 \\
0,05\end{array}$ & $\begin{array}{l}0,46 \\
0,13 \\
0,08 \\
0,06 \\
0,06 \\
0,03\end{array}$ & $\begin{array}{l}0,19 \\
0,20 \\
0,16 \\
0,14 \\
0,09 \\
0,13\end{array}$ & $\begin{array}{l}0,67 \\
0,04 \\
0,02 \\
0,06 \\
0,13\end{array}$ & $\begin{array}{l}7,43 \\
0,86 \\
0,42 \\
0,30 \\
0,26 \\
0,36\end{array}$ & $\begin{array}{l}3,29 \\
2,76 \\
2,24 \\
1,22 \\
0,70 \\
\\
0,97 \\
0,57 \\
0,16\end{array}$ & $\begin{array}{l}2,15 \\
5,11 \\
4,98 \\
2,25 \\
1,70 \\
0,90 \\
\end{array}$ & $\begin{array}{l}68,1 \\
19,45 \\
14,75 \\
12 \\
26,6 \\
\\
22,37 \\
11,62 \\
13,30 \\
9,63 \\
11,12 \\
7,5 \\
\end{array}$ & $\begin{array}{l}11 \\
8,5 \\
3,3 \\
2,3 \\
1,4 \\
33 \\
7,4 \\
3,1 \\
3,1 \\
2,3 \\
5 \\
\end{array}$ & $\begin{array}{c}14,11 \\
11,43 \\
11,03 \\
9 \\
6,68 \\
10,83 \\
6,85 \\
5,84 \\
2,71 \\
2,1 \\
1,4 \\
\end{array}$ & $\begin{array}{c}55 \\
14 \\
5 \\
3 \\
6 \\
\\
69,6 \\
12,5 \\
7,2 \\
11 \\
12,4 \\
25,7 \\
\end{array}$ \\
\hline
\end{tabular}

$V=S / T \times 100$ à pH7 et au pH du sol.

l'humification sur serpentines. La désaturation profonde sur les autres roches ne permet pas une évolution aussi favorable sur celles-ci (tableau V).

Au total les sols brunifiés, faiblement ocreux et à humus acide des serpentines s'opposent aux sols podzoliques plus ou moins humifères et aux podzols développés sur prasinites et gneiss acides (fig. 4).

\section{DISCUSSION ET CONCLUSION}

La forêt subalpine de ces 3 vallées du versant sud des Alpes pennines, en Vallée d'Aoste, s'articule autour de 3 essences forestières: le mélèze, l'épicéa, le pin cembro. Ces essences se répartissent de façon 


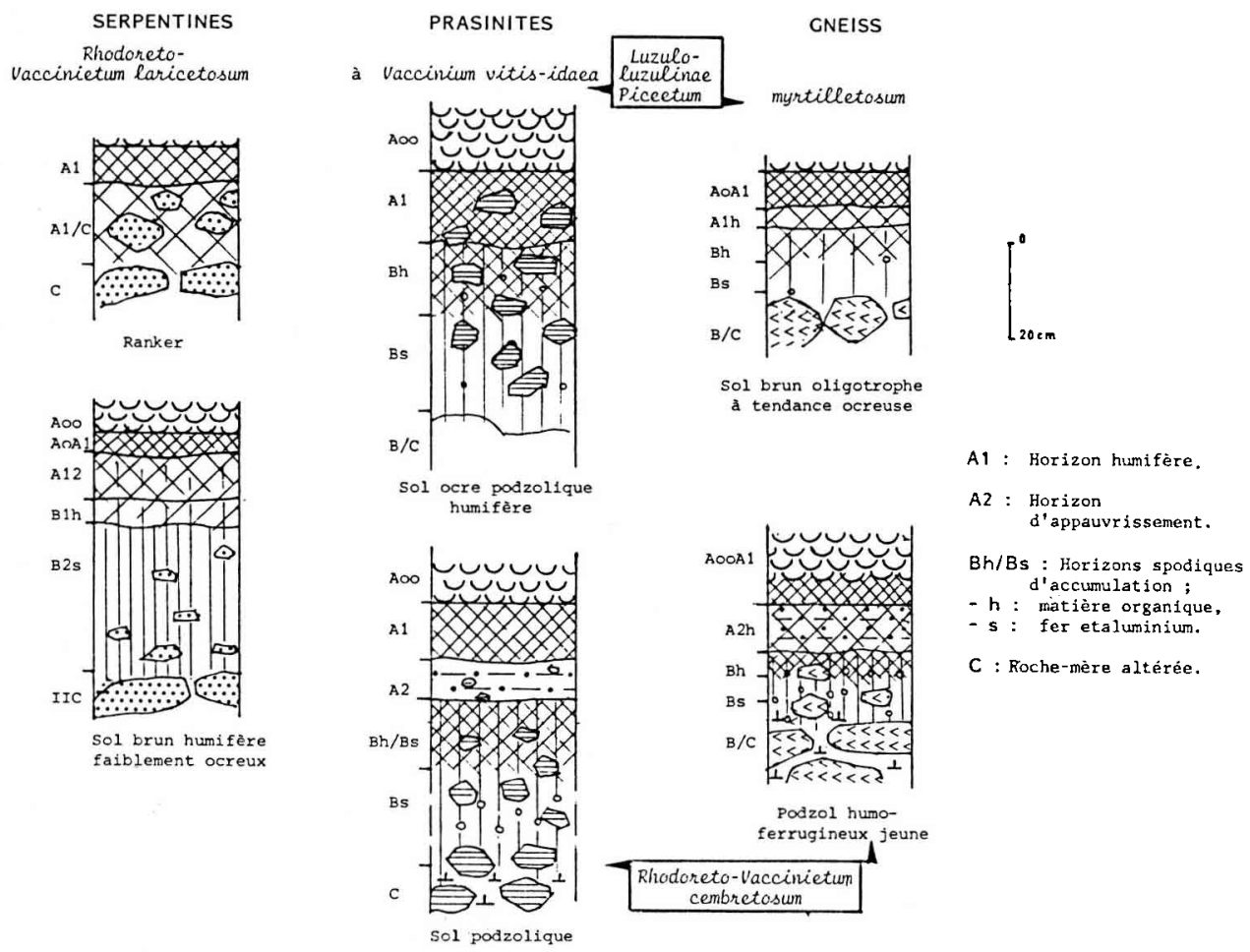

Fig. 4. Caractères morphologiques et nature des profils de la forêt subalpine sur serpentines, prasinites et gneiss en Val d'Aoste (Italie).

très diverse selon la roche mère qui module la composition et l'évolution de la forêt:

- 1) les rapports des ions de l'acidité (Ae) par rapport aux cations du complexe adsorbant (S) sont favorables aux espèces neutrophiles et surtout acidoclines sur serpentines (tableaux II et V). Tant sur prasinites que sur gneiss, les humus sont immédiatement favorables aux seules plantes acidophiles (tableaux III et V). L'acidification profonde limitée des ultrabasites ne permet pas une domination massive de ces espèces sur ce substrat, à l'inverse des autres roches;

-2) le mélèze trouve sur les fortes pentes des serpentines une persistance des conditions pédobiologiques primaires favorables à son installation et à son maintien grâce à une végétation ouverte, un sous-sol caillouteux, toujours bien aéré. L'humidité est suffisante et l'acidité jamais élevée en profondeur $(\mathrm{pH}>5)$, cette valeur constituant, selon Fourchy (1952), un seuil en dessous duquel, avec la podzolisation, le mélèze perd du terrain devant l'épicéa et le cembro. Le magnésium et le fer, toujours abondants, s'opposent à la podzolisation, de sorte que les conditions édaphiques restent favorables au maintien du mélèze; 
- 3) sur prasinites et gneiss, l'évolution biochimique des sols est celle de la podzolisation: matière organique abondante, acide, profondément incorporée et à faible vitesse de décomposition, basses valeurs $\mathrm{du} \mathrm{pH}$ liées à la dominance des ions de l'acidité, forte redistribution du fer et de l'aluminium. Ces phénomènes conjugués d'acidification et podzolisation croissante font que la pessière, vers le bas de l'étage, la cembraie vers le haut, supplantent progressivement le mélèze sur ces roches. L'humus brut n'est pas favorable à cette espèce et favorise surtout la germination de l'épicéa et du cembro au détriment du mélèze, aussi défavorisé par la densification végétale de surface;

- 4) l'absence de la pessière sur serpentinites peut s'expliquer par au moins 2 caractères: la faible acidité des sols, souvent limitée à l'humus, acidité qui disparaît en profondeur avec la saturation des sols par le magnésium; la sécheresse édaphique de surface trop forte, défavorable, en raison de la grande perméabilité des sols;

- 5) le pin cembro se comporte, dans le haut de l'étage, comme l'épicéa dans le bas. Les mêmes facteurs qui s'opposent au développement de cette espèce sur serpentines jouent également contre l'arolle. Le tapis des mousses acidophiles qui recouvre le sous-bois sur prasinites et gneiss est absent. Le rôle tampon des bryophytes, "particulièrement vis-à-vis de l'évaporation et de la transpiration (Lecointe, 1985)" est bien connu. Leur absence constitue un facteur aggravant de la sécheresse édaphique et crée des conditions défavorables à la germination de l'arolle. La réalisation d'écogramme du type de celui proposé par Auliztky (1963, dans Contini et Lavarello, 1982) fait apparaître la sécheresse comme agent de dégâts sur les cembraies où se rencontrent des espèces comme Calluna vulgaris, Vaccinium vitis-idaea, Jupinerus nana, très fréquentes sur serpentines. Les
$\mathrm{pH}$ supérieurs à 5,5 sont défavorables à la mycorhization, sauf pour quelques espèces liées au mélèze sur sols calcaires. Cette association intervient "dans l'adaptation des espèces aux conditions d'altitude (Contini et Lavarello, 1982)". Cette limite est vite dépassée, dès -10 à $-15 \mathrm{~cm}$ sous la rhodoraie sur serpentines. C'est sans doute à la conjonction de ces caractères, qui s'opposent aux conditions favorables au mélèze, qu'il faut attribuer l'absence de l'arolle;

- 6) on remarque aussi sur serpentinites l'absence du pin à crochets. Cette essence est capable de supporter une grande sécheresse édaphique, comme en Briançonnais (Cadel, 1980) ou en vallée de Ponton, au sud du Val d'Aoste (Vaccari, 1903). L'altération et l'argilisation accrue des sols serpentiniques (Verger, 1987 et 1989a) entretiennent à la fois une certaine humidité édaphique et une richesse cationique. Ce double effet favorise les essences moins frugales que le pin à crochets et principalement le mélèze.

En conclusion, dans le contexte climatique des vallées d'Aoste, les serpentines induisent, par leurs caractéristiques particulières, la répartition des essences forestières. Agissent-elles directement par leur forte teneur en magnésium ? Nous n'avons aucun élément permettant de l'affirmer. Par contre, de façon indirecte, en saturant le complexe adsorbant, le magnésium, mais aussi le fer, limitent ou empêchent l'acidification des sols, s'opposent au développement des mousses acidophiles et contribuent à la xéricité du milieu. Ces éléments vont à l'encontre de l'installation de l'épicéa et/ou du pin cembro.

\section{REMERCIEMENTS}

Je remercie MM. Cadel et Souchier, de l'université de Grenoble, pour leur aide et leurs conseils. 


\section{RÉFÉRENCES}

Bartoli Ch (1966) Etudes écologiques sur les associations forestières de la Haute-Maurienne. Ann Sci For (Paris) XXIII, 3, 433-749

Boillot $\mathrm{G}$ (1987) Les fonds sous-marins du troisième type. Recherche, 19 3, 1 294-1 303

Braun-Blanquet J (1964) Pflanzensoziologie. Springer Verlag, Vienne

Braun-Blanquet J, Sissingh C, Vlieger J (1939) Prodomus der Pflanzengenellschaften, fasc 6, Klasse der Vaccinio-Piceatea. Comité Int. du Prodome Phytosocial.

Braun-Blanquet J, Palmann H, Bach R (1954) Vegetation und Böden der Waldund Zwergstranchgesellschaften (Vaccinio-Piceetalia). In: Résultats des recherches scientifiques entreprises au Parc National Suisse. IV, 1-198

Cadel G (1980) Séries de végétation et sols du subalpin briançonnais sur roches mères silico-alumineuses. Comparaison avec la Maurienne et la Tarentaise. Sci Sol 4, 249-264

Contini L, Lavarello $Y$ (1982) Le Pin cembro: répartition, écologie, sylviculture et production. INRA, Paris

Fourchy $P$ (1952) Ecologie du mélèze, particulièrement dans les Alpes françaises. Ann. Ecole Nat Eaux et Forêts 13,1, 1-37

Gams H (1950) Guide pour la $78^{e}$ session extraordinaire de la Société Botanique Française. Excursion internationale. "Coupe Botanique des Alpes". $57 \mathrm{p}$.

Gensac P (1964) Les Pessières de Tarentaise. Etude phytogéographique et pédologique. Doc Carte Vég Alpes II, 119-155

Jeanroy $E$ (1983) Diagnostic des formes du fer dans les pédogénèses tempérées. Evaluation par les réactifs chimiques d'extraction et apports de la spectrométrie Mossbauer. Thèse de doctorat de l'université de Nancy-I

Lecointe A (1985) Les difficultés dans l'utilisation des Bryophytes en typologie forestière: exemple du Pays d'Auge (Normandie). Col Phyt Phytosociologie et Forestière XIV, Nancy, 629-653

Lyabel C (1962) Le problème forestier en Vallée d'Aoste. Flambeau 3, 63-81

Ozenda P (1981) Végétation des Alpes sudoccidentales. Editions du CNRS

Ozenda P (1983) La végétation de l'arc alpin. Comité européen pour la sauvegarde de la nature et des ressources naturelles. Strasbourg

Penel M (1979) Caractérisation physicochimique et classification des humus forestiers acides en relation avec la végétation et ses exigences écologiques. Thèse de doctorat de spécialité de l'université de Nancy-I

Rey P (1960) Essai de phytocinétique biogéographique. Thèse Etat, CNRS, Toulouse

Richard JL (1985) Observations sur la sociologie et l'écologie de Carex fimbriata Schkuhr dans les Alpes. Bot Helv 9512, 157164

Richard L, Tonnel A (1987) Contribution à l'étude des vallées internes des Alpes occidentales. Première partie: originalité du milieu et quelques conséquences biologiques. Doct Cart Ecol XXX, 113-136

Robin AM (1979) Genèse et évolution des sols podzolisés sur affleurements sableux du bassin parisien. Thèse de doctorat d'Etat de l'université de Nancy-l

Rouiller J (1981) Analyses des sols. Techniques de laboratoire. Centre Ped Biol 32, CNRS, Nancy

Souchier B (1971) Evolution des sols sur roches cristallines à l'étage montagnard (Vosges). Thèse de doctorat d'Etat de l'université de Nancy

Vaccari L (1903) La flore de la serpentine, du calcaire et du gneiss dans les Alpes graies orientales. Bull Soc Flore Valdot 2

Verger JP (1983) Contribution à la connaissance d'un groupement alpin climacique original sur serpentines: le Caricetum fimbriatae. Phytosociologie et pédologie CR Acad Sci Paris 296, 775-778

Verger JP (1987) Végétation et pédogenèse sur roches vertes et gneiss acide dans une séquence altitudinale montagnard-alpin en Val d'Aoste (Italie). Essai de synthèse écologique. Thèse de doctorat d'Etat de l'université de Grenoble

Verger JP (1989a) Evolution granulométrique des sols sur roches ultrabasique, basique et acide en Val d'Aoste (Italie). Conséquences sur la végétation. Sci Sol 1 (sous-presse)

Verger JP (1989b) Rôle des ions de l'acidité dans la répartition des espèces végétales. Application à quelques espèces de l'étage alpin. Bull Ecol (à paraître) 